\section{O Preço e o Valor do Tratamento da Osteoporose}

A PREOCUPAC̄ão COM OS CUSTOS dos tratamentos de enfermidades crônicas tem ocupado um capítulo importante da política de saúde brasileira. Desde o governo anterior e, ainda mais no presente, o uso de medicamentos genéricos tem sido defendido e estimulado.

Entende-se por medicamentos genéricos aqueles que são disponibilizados no mercado, após o período de expiração das patentes internacionais dos produtos originais, e que apresentem estudos de bioequivalência e biodisponibilidade que atestem eficácia e segurança comparáveis às do produto original.

Contudo, a plena compreensão desses detalhes acima é, freqüentemente, ofuscada pela nossa realidade econômica que abre espaços para medicamentos denominados "similares" que, para fazer jus a este título ostentam, unicamente, alguns papéis e carimbos versando sobre sua regularidade fiscal e tributária, além de certificados de origem duvidosa dos sais utilizados. Não dispõem, lamentavelmente, de ensaios de farmacodinâmica e, muito menos, de estudos clínicos que confirmem sua eficácia e segurança.

Estudos (1-6) têm avaliado e comparado a eficácia e a segurança de medicamentos denominados "similares", deixando claro que, em muitos casos, a equivalência esperada não é a regra e que, sobretudo, riscos adicionais são esperados com o emprego desses.

Dentre o grupo de doenças crônicas onde medicamentos ditos similares têm sido disponibilizados, a osteoporose se destaca pela sua prevalência e notórias repercussões clínicas. Novos agentes antireabsortivos demonstraram, em estudos clínicos de longo prazo, desenhados e realizados segundo elevados níveis da hierarquia científica, serem capazes de produzir ganhos de densidade mineral óssea e, ainda mais importante, reduzir a ocorrência cumulativa de fraturas quando comparados a grupos controle (7-9).

Dentre esses medicamentos, o alendronato de sódio (marca original: Fosamax ${ }^{\circledR}$, do laboratório Merck Sharp \& Dohme), substância do grupo dos bisfosfonatos, se destaca pelo tempo de experiência clínica já acumulada e, até por isso, pelo número de "similares" já introduzidos no mercado brasileiro e latino-americano.

Os bisfosfonatos são, por natureza, pobremente absorvidos quando administrados por via oral. Ensaios clínicos realizados demonstraram que, mesmo após jejum de 2 horas, seu perfil de absorção oral era significativamente prejudicado, sendo necessário jejum de, no mínimo, 8 horas ou mais além de sua manutenção por, pelo menos, 30 minutos para que sua absorção e efeitos sejam os esperados (10).

A biodisponibilidade e absorção dos bisfosfonatos podem depender da sua fabricação, formulação e demais características do comprimido, em diferentes níveis. A presença de comida ou outras substâncias (além da água) reduz a biodisponibilidade dos bisfosfonatos.

Também aprendemos, com os estudos clínicos, que os bisfosfonatos orais podem aumentar o risco de eventos adversos ao nível do esôfago, o que faz das condições de administração, desintegração, dissolução e absorção características extremamente importantes para que sua eficácia seja

\section{cartas ao editor}

Sergio Ragi Eis
CEDOES - Diagnóstico e Pesquisa da Osteoporose 
garantida e, ainda mais, para que os pacientes não sejam expostos a riscos desnecessários.

Segundo a Organização Mundial da Saúde (5), “...para formas genéricas orais, estudos de bioequivalência contra $\mathrm{o}$ produto original são requeridos, exceto quando comparações de dissolução in vitro forem comprovadamente suficientes".

Recentemente, tivemos a oportunidade de participar de um estudo (11) que comparou o tempo de desintegração de 13 "similares" disponíveis em vários países latino-americanos, dentre os quais dois em comercialização no Brasil.

Define-se desintegração como o processo físico através do qual um comprimido de fragmenta em finas partículas. Neste estudo, o tempo de desintegração foi medido, in vitro, através de um procedimento padrão da "Farmacopéia Norte-americana", aprovado pelo FDA (Food and Drugs Administration), realizado em solução aquosa padrão à $37^{\circ} \mathrm{C}$, utilizando-se várias amostras de diferentes lotes de cada cópia avaliada.

O tempo médio de desintegração do medicamento original, medido em 4 dias diferentes e em lotes distintos do medicamento foi de $86( \pm 11,8)$ segundos. Para uma das cópias brasileiras (Cópia 1) observou-se tempo de desintegração médio 2,5 vezes menor e, para a outra (Cópia 2), o tempo médio de desintegração foi até 22 vezes maior.

Considerando-se as características abordadas de absorção, eficácia e segurança clínica do alendronato de sódio, essas diferenças observadas (Tabela 1) podem determinar, em princípio:

1. Para a Cópia $\mathbf{1}$, que teve seu processo de desintegração ao redor de $32( \pm 4,7)$ segundos, pode-se imaginar que sua defragmentação pode ocorrer ainda durante o trajeto do comprimido ao nível do esôfago. Evidentemente que os riscos de irritação da mucosa esofágica, erosão e, até mesmo, úlceras será superior, além de poder prejudicar o perfil de absorção do medicamento;

2. Para a Cópia 2, com perfil de desintegração médio de $21( \pm 0,9)$ minutos; chegando até a 46,5 minutos; uma vez que a absorção dos bisfosfonatos se dá ao nível da porção proximal do intestino delgado, pode-se inferir que sua absorção estará, em muito, prejudicada, uma vez que o princípio ativo não estará em condições de ser absorvido durante sua passagem na porção do intestino onde isso ocorre. Além disto os 30 minutos de jejum recomendados, após a ingestão do comprimido, seguramente não seriam suficientes.
Tabela 1. Diferenças no perfil de desintegração entre Fosamax®, Cópia 1 e Cópia 2.

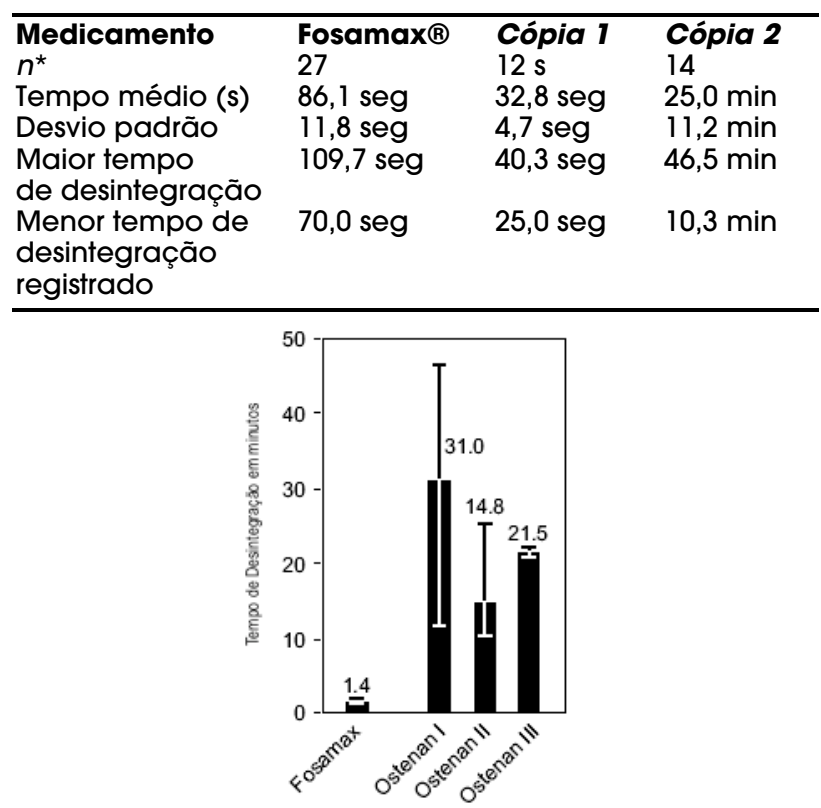

Gráfico 1. Comparação do tempo de desintegração do Fosamax® vs. Cópia 2.

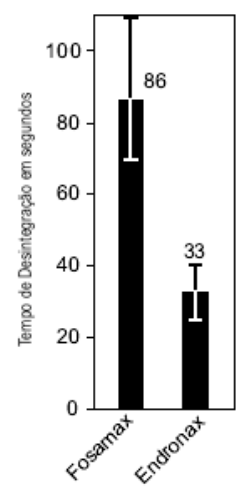

Gráfico 2. Comparação do tempo de desintegração do Fosamax® vs. Cópia 1.

Muitas são as outras características dos medicamentos que podem interferir nas suas equivalências em relação aos produtos originais. A forma, revestimento $\mathrm{e}$ desenho dos comprimidos e cápsulas, o excipiente e outras substâncias empregadas na formulação, a pureza (origem) do sal ativo, quantidade do princípio ativo no comprimido, dentre outros.

Tendo isso em mente e baseando-se nos resultados apresentados $\mathrm{e}$, ainda mais, em atenção às recomendações da Organização Mundial da Saúde para medicamentos genéricos (5), torna-se imperativo o estabelecimento de exigências específicas e rígidas para que medicamentos possam ser considerados "equivalentes" aos originais.

Arq Bras Endocrinol Metab vol 48 n 6 Dezembro 2004 
Ao médico cabe reconhecer que uma prescrição é, em verdade, um aval ao medicamento prescrito quanto à sua eficácia e, não menos importante, sua segurança. O evento adverso ou a não eficácia de um medicamento, sob esse ponto de vista, deverão ser considerados iatrogenias. Assim, a exigência de estudos de bioequivalência e, até mesmo, de evidências clínicas de eficácia e segurança, deve começar por quem assume a responsabilidade por suas prescrições - nós médicos.

Aos pacientes, cabe o reconhecimento de que o valor do tratamento se mede pelo custo do medicamento, aliado a sua eficácia e segurança. A observação, simplista, de que medicamentos são mais baratos sem o cuidado de se observar seu nível de eficácia (e segurança) clínica pode conduzir a tratamentos muito mais caros, mesmo com medicamentos de preços mais baratos. A velha teoria de que muitas vezes o barato sai caro se aplica.

Aos profissionais de farmácia cabe um maior compromisso com tais questões técnicas e evitarem o procedimento, ainda freqüente no Brasil, de estimular o uso de medicamentos apenas pelo preço, não dando a devida atenção às evidências científicas, muitas vezes em detrimento do medicamento originalmente prescrito.

Reconhecer a necessidade de se baixar custos dos medicamentos para permitir acesso aos tratamentos é tarefa de todos, mas a atenção aos princípios científicos que norteiam a prática não pode ser ofuscada. $\mathrm{O}$ custo do tratamento de uma fratura osteoporótica pode ser, de longe, muito maior do que o do tratamento da osteoporose. Se faltarem recursos para o custeio de tratamentos comprovadamente eficazes, estes também não serão disponíveis para o tratamento das fraturas não prevenidas e, ainda mais, das iatrogenias produzidas por medicamentos de segurança e eficácia duvidosas.

\section{REFERÊNCIAS}

1. Bailey RT Jr, Bonavina L, McChesney L, Spires KJ, Muilenberg MI, McGill JE, et al. Factors influencing the transit of a gelatin capsule in the esophagus. Drug Intell Clin Pharm 1987;21:282-5
2. Channer KS, Virjee JP. The effect of formulation on oesophageal transit. J Pharm Pharmacol 1985;37:1269.

3. Perkins $A C$, Wilson $C G$, Blackshaw PE, Vincent RM, Dansereau RJ, Juhlin KD, et al. Impaired esophageal transit of capsule versus tablet formulations in the elderly. Gut 1994;35:1363-7.

4. Marvola M, Vahervuo K, Sothmann A, Marttila E, Rajaniemi M. Development of a method for study of the tendency of drug products to adhere to the esophagus. J Pharm Sci 1982;71:975-7.

5. Videau J-Y. Making medicines safe. Bull World Health $2001 ; 79: 87$.

6. Rägo L. Ensuring access to drug products that are of acceptable quality. WHO Pilot Procurement Quality and Sourcing prequalification') project. WHO Web Site: http://www.who.int/medicines/organization/par/b riefing/ 9prequalification.ppt September 4, 2003.

7. Liberman UA, Weiss SR, Broll J, Minne HW, Quan H, Bell $\mathrm{NH}$, et al. Effect of oral alendronate on bone mineral density and the incidence of fractures in postmenopausal osteoporosis. The Alendronate Phase III Osteoporosis Treatment Study Group. N Engl J Med 1995;333: 1437-43.

8. Devogelaer JP, Broll H, Correa-Rotter R, Cumming DC, De Deuxchaisnes $C N$, Geusens $P$, et al. Oral alendronate induces progressive increases in bone mass of the spine, hip, and total body over 3 years in postmenopausal women with osteoporosis. Bone 1996; 18:141-50.

9. Tonino RP, Meunier PJ, Emkey R, Rodriguez-Portales JA, Menkes CJ, Wasnich RD, et al. Skeletal benefits of alendronate: 7 year treatment of postmenopausal osteoporotic women. J Clin Endocrinol Metab 200 0;85:310915.

10. Fosamax Prescribing Information. Physicians' desk ref erence, 55th ed. Montvale, NJ: Medical Economics Co; 2001:1930-6.

11. Epstein S, Cryer B, Ragi S, Zanchetta JR, Walliser J, Chow J, et al. Disintegration/ dissolution profiles of copies of Fosamax (alendronate). Curr Med Res Opin 2003; 19(8):781-89.

Endereço para correspondência:

Sergio Ragi Eis

Rua João da Silva Abreu 78

Praia do Canto

29055-450 - Vitória, ES 